\title{
Damping influence on the critical velocity and response characteristics of structurally pre-stressed beam subjected to traveling harmonic load
}

\author{
B. Omolofe $\mathrm{a}^{\mathrm{a}, *}$, T. O. Awodola ${ }^{\mathrm{a}}$, T. O. Adeloye ${ }^{\mathrm{b}}$ \\ ${ }^{a}$ Department of Mathematical Sciences, School of Sciences, Federal University of Technology, P. M. B 704, Akure Ondo State, Nigeria. \\ ${ }^{b}$ Department of Mathematics, Faculty of Basic Sciences, Nigeria Maritime University, Okerenkoko, Delta State, Nigeria.
}

\begin{abstract}
In this present study, the response characteristics of a flexible member carrying harmonic moving load are investigated. The beam is assumed to be of uniform cross section and has simple support at both ends. The moving concentrated force is assumed to move with constant velocity type of motion. A versatile mathematical approximation technique often used in structural mechanics called assumed mode method is in first instance used to treat the fourth order partial differential equation governing the motion of the slender member to obtain a sequence of second order ordinary differential equations. Integral transform method is further used to treat this sequence of differential equations describing the motion of the beam-load system. Various results in plotted curves show that, the presence of the vital structural parameters such as the axial force $\mathrm{N}$, rotatory inertia correction factor $r^{0}$, the foundation modulus $F_{0}$, and the shear modulus $G_{0}$, significantly enhances the stability of the beam when under the action of moving load. Dynamic effects of these parameters on the critical speed of the dynamical system are carefully studied. It is found that as the values of these parameters increase, the critical speed also increases. Thereby reducing the risk of resonance and thus the safety of the occupant of this structural member is guaranteed.
\end{abstract}

Keywords: Response characteristics, flexural member, harmonic load, critical speed, resonance, foundation stiffness, assumed mode, concentrated force.

2010 MSC: 82D40, 74F15, 74H45, 74K20.

(C)2018 All rights reserved.

\section{Introduction}

The amplitude of the dynamic deflection and vibration control of beam-like structures carrying moving masses have long been an exciting subject and of great fundamental importance to many researchers. In fact, it is one of the most important subjects in the area of structural dynamics and vibration control. This is due largely to its enormous applications in engineering sciences. In particular, tracks on which vehicle or train travels, fluid-conveying pipe system, beams under the actions of pressure waves and shafts

\footnotetext{
${ }^{*}$ Corresponding author

Email addresses: babatope_omolofe@yahoo.com (B. Omolofe), toawodola@yahoo.com (T. O. Awodola), adeyel@yahoo.com (T. O. Adeloye)
}

doi: $10.22436 / \mathrm{mns} .03 .01 .03$

Received: 2017-10-16 Revised: 2018-04-02 Accepted: 2018-04-06 
or machining operations which requires axial motions can all be model as continuum systems continuously supporting or carrying moving sub-systems. Bridges, railway bridges, cranes, cable ways, tunnels, and pipes are the typical structural examples of the structure to be designed to support moving masses and loads.

Consequently, there is a large volume of literature devoted to moving load problems in the past few years see for instance $[2,4-6,9,10,14,19-22,24,28,29,31,32,34-38,40]$ and the references therein. The theoretical and experimental studies of the moving load problems have shown that moving loads may be divided into three categories namely: moving oscillator, moving mass, and moving force. Vibrations of beams due to moving oscillators are studied in [14, 17, 23, 33, 39], vibrations of beams due to a moving mass are investigated in $[3,4,13,16,25,36,41]$, and vibration of beams due to moving force have been considered in $[1,8,15,18,27,40]$. Moving load problem with or without elastic foundation has been extensively studied and so many other aspects of the moving load problem have also been considerably explored [7, 11, 12, 21, 26, 30, 34].

In this present study an approximate analytical solution of the transverse response of a simply supported Rayleigh beam resting on variable two-parameter elastic sub-grade and carrying harmonic variable load traveling with constant velocity type of motion is obtained. Effects of internal and external damping on the dynamic characteristics of a beam-like structural member carrying moving load are well studied. Effects of these and some other vital structural parameters on the critical speed of this vibrating system will also be established.

\section{Mathematical formulation}

Consider a structurally prestressed Rayleigh beam under the actions of traveling load of mass M. The span length of the beam is finite and the mass is assumed to travel along the beam with a constant velocity. Considering damping effects, the transverse displacement in terms of traveling time $t$ and the spatial coordinate is prescribed by the fourth order partial differential equation given by

$$
\begin{aligned}
E I \frac{\partial^{4} W(x, t)}{\partial x^{4}} & -N \frac{\partial^{2} W(x, t)}{\partial x^{2}}+\mu \frac{\partial^{2} W(x, t)}{\partial t^{2}}-\mu r^{0} \frac{\partial^{4} W(x, t)}{\partial x^{2} \partial t^{2}} \\
& +F(x) W(x, t)+D_{e} \frac{\partial W(x, t)}{\partial t}+D_{i} \frac{\partial^{5} W(x, t)}{\partial t \partial x^{4}}-\frac{\partial^{4}}{\partial}\left[G(x) \frac{\partial W(x, t)}{\partial x}\right]=P(x, t) .
\end{aligned}
$$

Where EI is the flexural rigidity of the beam, $\mu$ is the mass per unit length of the beam, and $F(x)$ and $G(x)$ are the variable foundation stiffness and shear modulus, respectively. $W(x)$ is the deflection of the beam at point $x$ and time $t . P(x, t)$ denotes the traveling load. A prime denotes differentiation with respect to position coordinate $x$ and an over-dot represents differentiation with respect to time $t$. The external damping $\mathrm{D}_{e}$ and internal damping $\mathrm{D}_{i}$ of the beam are taken to be proportional to the mass and stiffness of the beam respectively and are given as

$$
D_{e}=\eta_{e} \mu, \quad D_{i}=\eta_{i} E I,
$$

where $\eta_{e}$ and $\eta_{i}$ are the constants of proportionality.

In this study, it is assumed that the load function $P(x, t)$ is given in the form

$$
P(x, t)=h(x) \cos w t,
$$

where $h(x)$ is an arbitrary deterministic distributed load.

Now, considering the action of a concentrated harmonic force $H(t)$ at a position, $x=u t$. The load $P(x, t)$ can be written as

$$
P(x, t)=H_{0} \cos w t \delta(x-u t),
$$


$\delta(\cdot)$ is the well-known Dirac delta function with the property

$$
\int_{a}^{b} \delta(x-k) f(x) d x= \begin{cases}0, & \text { for } k<a<b \\ f(k), & \text { for } a<k<b \\ 0, & \text { for } a<b<k\end{cases}
$$

The variable foundation stiffness and shear modulus are respectively taken to be

$$
\mathrm{F}(\mathrm{x})=\mathrm{F}_{0}\left(4 x-3 x^{2}+x^{3}\right)
$$

and

$$
\mathrm{G}(\mathrm{x})=\mathrm{G}_{0}\left(12-13 \mathrm{x}+6 \mathrm{x}^{2}-\mathrm{x}^{3}\right) .
$$

It is remarked here that the beam under consideration is assumed to have simple support at both ends $x=0$ and $x=L$. Thus boundary conditions are given as

$$
W(0, t)=0=W(L, t), \frac{\partial W(0, t)}{\partial x}=0=\frac{\partial^{2} W(L, t)}{\partial x^{2}}
$$

and the initial conditions is given as

$$
W(0, t)=0=\frac{\partial W(x, 0)}{\partial t} .
$$

Substituting equation (2.2) into (2.1) we have

$$
\begin{aligned}
E I \frac{\partial^{4} W(x, t)}{\partial x^{4}} & -N \frac{\partial^{2} W(x, t)}{\partial x^{2}}+\mu \frac{\partial^{2} W(x, t)}{\partial t^{2}}-\mu r^{0} \frac{\partial^{4} W(x, t)}{\partial x^{2} \partial t^{2}}+F(x) W(x, t) \\
& +D_{e} \frac{\partial W(x, t)}{\partial t}+D_{i} \frac{\partial^{5} W(x, t)}{\partial t \partial x^{4}}-\frac{\partial}{\partial x}\left[G(x) \frac{\partial W(x, t)}{\partial x}\right]=H_{0} \cos w t \delta(x-u t) .
\end{aligned}
$$

Equation (2.4) is the fourth order partial differential equation governing the flexural motion of the Structurally prestressed Rayleigh beam.

\section{Solution procedures}

To find an approximate solution of the boundary-initial-value problem (2.1), an assumed mode method is employed. By this method, the $\mathrm{j}^{\text {th }}$ term approximate solution of (2.4) is sought in the form.

$$
W_{j}(x, t)=\sum_{m=1}^{\infty} Z_{m}(t) u_{m}(x),
$$

where $Z_{m}(t)$ are coordinates in modal space and $U_{m}(x)$ are the normal modes of vibration written as

$$
U_{m}(x)=\sin \frac{\lambda_{m} x}{L}+A_{m} \cos \frac{\lambda_{m} x}{L}+B_{m} \sinh \frac{\lambda_{m} x}{L}+C_{m} \cosh \frac{\lambda_{m} x}{L} .
$$

It can be shown that, for a structural member having simple supports at ends $x=0$ and $x=L$ equation (3.1) in view of equation (3.2) can be written as

$$
W_{j}(x, t)=\sum_{m=1}^{\infty} Z_{m}(t) \sin \frac{m \pi x}{L} .
$$

Substituting equation (3.3) into the governing equation (2.4), one obtains

$$
E I\left(\frac{m \pi}{L}\right)^{4} \sum_{m=1}^{\infty} Z_{m}(t) \sin \frac{m \pi x}{L}+N\left(\frac{m \pi}{L}\right)^{2} \sum_{m=1}^{\infty} Z_{m}(t) \sin \frac{m \pi x}{L}+\mu \sum_{m=1}^{\infty} \ddot{Z}_{m}(t) \sin \frac{m \pi x}{L}
$$




$$
\begin{aligned}
& +\mu r^{0}\left(\frac{m \pi}{L}\right)^{2} \sum_{m=1}^{\infty} \ddot{Z}_{m}(t) \sin \frac{m \pi x}{L}+F_{0}\left(4 x-3 x^{2}+x^{3}\right) \sum_{m=1}^{\infty} Z_{m}(t) \sin \frac{m \pi x}{L}+D_{e} \sum_{m=1}^{\infty} \dot{Z}_{m}(t) \sin \frac{m \pi x}{L} \\
& +D_{i}\left(\frac{m \pi}{L}\right)^{4} \sum_{m=1}^{\infty} \dot{Z}_{m}(t) \sin \frac{m \pi x}{L}+G_{0}\left(12-13 x+6 x^{2}\right)\left(\frac{m \pi}{L}\right)^{2} \sum_{m=1}^{\infty} Z_{m}(t) \sin \frac{m \pi x}{L} \\
& +G_{0}\left(13-12 x+3 x^{2}\right) \frac{m \pi}{L} \sum_{m=1}^{\infty} Z_{m}(t) \cos \frac{m \pi x}{L}=H_{0} \cos w t \delta(x-u t),
\end{aligned}
$$

which after some re-arrangements gives

$$
\begin{aligned}
& \sum_{m=1}^{\infty}\left\{\left(\mu \sin \frac{m \pi x}{L}+\mu r^{0}\left(\frac{m \pi}{L}\right)^{2} \sin \frac{m \pi x}{L}\right) \ddot{Z}_{m}(t)+\left(D_{e} \sin \frac{m \pi x}{L}+D_{i}\left(\frac{m \pi}{L}\right)^{4} \sin \frac{m \pi x}{L}\right) \dot{Z}_{m}(t)\right. \\
& \quad+\left(E I\left(\frac{m \pi}{L}\right)^{4} \sin \frac{m \pi x}{L}+N\left(\frac{m \pi}{L}\right)^{2} \sin \frac{m \pi x}{L}+F_{0}\left(4 x-3 x^{2}+x^{3}\right) \sin \frac{m \pi x}{L}\right. \\
& \left.\left.\quad+G_{0}\left(12-13 x+6 x^{2}-x^{3}\right)\left(\frac{m \pi}{L}\right)^{2} \sin \frac{m \pi x}{L}+G_{0}\left(12-13 x+6 x^{2}-x^{3}\right) \frac{m \pi x}{L} \sin \frac{m \pi x}{L}\right) Z_{m}(t)\right\} \\
& \quad-H_{0} \cos w t \delta(x-u t)=0 .
\end{aligned}
$$

In order to determine the expression for $Z_{m}(t)$, it is required that the expression on the LHS of equation (3.4) be orthogonal to the function $\sin \frac{k \pi x}{L}$. Thus, multiplying equation (3.4) by $\sin \frac{k \pi x}{L}$ and integrating from $x=0$ to $x=L$ leads to

$$
\begin{aligned}
& \int_{0}^{L}\left[\sum _ { m = 1 } ^ { \infty } \left\{\left(\mu \sin \frac{m \pi x}{L}+\mu r^{0}\left(\frac{m \pi}{L}\right)^{2} \sin \frac{m \pi x}{L}\right) \ddot{Z}_{m}(t)+\left(D_{e} \sin \frac{m \pi x}{L}+D_{i}\left(\frac{m \pi}{L}\right)^{4} \sin \frac{m \pi x}{L}\right) \dot{Z}_{m}(t)\right.\right. \\
& +\left(E I\left(\frac{m \pi}{L}\right)^{4} \sin \frac{m \pi x}{L}+N\left(\frac{m \pi}{L}\right)^{2} \sin \frac{m \pi x}{L}+F_{0}\left(4 x-3 x^{2}+x^{3}\right) \sin \frac{m \pi x}{L}\right. \\
& \left.\left.\quad+G_{0}\left(12-13 x+6 x^{2}-x^{3}\right)\left(\frac{m \pi}{L}\right)^{2} \sin \frac{m \pi x}{L}+G_{0}\left(12-13 x+6 x^{2}-x^{3}\right) \frac{m \pi x}{L} \sin \frac{m \pi x}{L}\right) Z_{m}(t)\right\} \\
& \left.-H_{0} \cos w t \delta(x-u t)\right] \sin \frac{k \pi x}{L} d x=0,
\end{aligned}
$$

which after some simplifications yields

$$
\begin{aligned}
\sum_{m=1}^{\infty} & \left\{\left(\vartheta_{1}+\vartheta_{2}\right) \ddot{Z}_{m}(t)+\left(\vartheta_{3}+\vartheta_{4}\right) \dot{Z}_{m}(t)+\left(\vartheta_{5}+\vartheta_{6}+\vartheta_{7}+\vartheta_{8}+\vartheta_{9}\right) Z_{m}(t)\right\} \\
= & \int_{0}^{L} H_{0} \cos w t \delta(x-u t) \sin \frac{k \pi x}{L} d x
\end{aligned}
$$

where

$$
\begin{array}{ll}
\vartheta_{1}=\mu \int_{0}^{\mathrm{L}} \sin \frac{\mathrm{m} \pi x}{\mathrm{~L}} \sin \frac{k \pi x}{\mathrm{~L}} \mathrm{~d} x, & \vartheta_{2}=\mu \mathrm{r}^{0}\left(\frac{\mathrm{m} \pi}{\mathrm{L}}\right)^{2} \int_{0}^{\mathrm{L}} \sin \frac{\mathrm{m} \pi x}{\mathrm{~L}} \sin \frac{k \pi x}{\mathrm{~L}} \mathrm{dx}, \\
\vartheta_{3}=\mathrm{D}_{1} \int_{0}^{\mathrm{L}} \sin \frac{\mathrm{m} \pi x}{\mathrm{~L}} \sin \frac{k \pi x}{\mathrm{~L}} \mathrm{dx}, & \vartheta_{4}=\mathrm{D}_{2}\left(\frac{\mathrm{m} \pi}{\mathrm{L}}\right)^{4} \int_{0}^{\mathrm{L}} \sin \frac{\mathrm{m} \pi x}{\mathrm{~L}} \sin \frac{k \pi x}{\mathrm{~L}} \mathrm{dx}, \\
\vartheta_{5}=\mathrm{EI}\left(\frac{\mathrm{m} \pi}{\mathrm{L}}\right)^{4} \int_{0}^{\mathrm{L}} \sin \frac{\mathrm{m} \pi x}{\mathrm{~L}} \sin \frac{\mathrm{k} \pi x}{\mathrm{~L}} \mathrm{dx}, & \vartheta_{6}=\mathrm{N}\left(\frac{\mathrm{m} \pi}{\mathrm{L}}\right)^{2} \int_{0}^{\mathrm{L}} \sin \frac{\mathrm{m} \pi x}{\mathrm{~L}} \sin \frac{k \pi x}{\mathrm{~L}} \mathrm{dx},
\end{array}
$$




$$
\begin{aligned}
& \vartheta_{7}=\int_{0}^{L} F_{0}\left(4 x-3 x^{2}+x^{3}\right) \sin \frac{m \pi x}{L} \sin \frac{k \pi x}{L} d x, \quad \vartheta_{8}=\left(\frac{m \pi}{L}\right)^{2} \int_{0}^{L} G_{0}\left(12-13 x+6 x^{2}\right) \sin \frac{m \pi x}{L} \sin \frac{k \pi x}{L} d x, \\
& \vartheta_{9}=\frac{m \pi}{L} \int_{0}^{L} G_{0}\left(13-12 x+3 x^{2}\right) \cos \frac{m \pi x}{L} \sin \frac{k \pi x}{L} d x .
\end{aligned}
$$

Noting property (2.2) and considering the $\mathrm{m}^{\text {th }}$ particle of the vibrating system, equation (3.5) can then be written after some rearrangements and simplifications as

$$
\ddot{Z}_{m}(t)+Q_{1} Z_{m}(t)+Q_{2} Z_{m}(t)=\frac{Q_{4}}{2}[\sin \alpha t-\sin \beta t],
$$

where,

$$
\mathrm{Q}_{1}=\frac{\vartheta_{3}+\vartheta_{4}}{\vartheta_{1}+\vartheta_{2}}, \mathrm{Q}_{2}=\frac{\vartheta_{5}+\vartheta_{6} \vartheta_{7}+\vartheta_{8} \vartheta_{9}}{\vartheta_{1}+\vartheta_{2}}, \mathrm{Q}_{3}=\vartheta_{1}+\vartheta_{2}, \mathrm{Q}_{4}=\frac{\mathrm{H}_{0}}{\mathrm{Q}_{3}}, \alpha=\omega+\frac{\mathrm{k} \pi \mathrm{u}}{\mathrm{L}}, \beta=\omega-\frac{\mathrm{k} \pi \mathrm{u}}{\mathrm{L}} .
$$

To obtain solution of the second order ordinary differential equation (3.6) above, we subjected it to a Laplace transform defined as

$$
\dot{\sim}=\int_{0}^{\infty}(\cdot) e^{-s t} \mathrm{dt}
$$

where $s$ is the Laplace parameter. Invoking the initial condition (2.3), one obtains the simple algebraic expression given as

$$
Z_{m}(s)=\frac{\frac{Q_{4}}{2}\left[\frac{\alpha}{s^{2}+\alpha^{2}}-\frac{\beta}{s^{2}+\beta^{2}}\right]}{s^{2}+s Q_{1}+Q_{2}},
$$

which is further simplified to give

$$
Z_{m}(s)=\frac{Q_{4}}{2\left(b_{1}-b_{2}\right)}\left\{\frac{\alpha}{s^{2}+\alpha^{2}} \cdot \frac{1}{s-b_{1}}-\frac{\alpha}{s^{2}+\alpha^{2}} \cdot \frac{1}{s-b_{2}}-\frac{\beta}{s^{2}+\beta^{2}} \cdot \frac{1}{s-b_{1}}+\frac{\beta}{s^{2}+\beta^{2}} \cdot \frac{1}{s-b_{2}}\right\},
$$

where

$$
\mathrm{b}_{1}=\frac{-\mathrm{Q}_{1}+\sqrt{\mathrm{Q}_{1}^{2}-4 \mathrm{Q}_{2}}}{2}, \mathrm{~b}_{2}=\frac{-\mathrm{Q}_{1}-\sqrt{\mathrm{Q}_{1}^{2}-4 \mathrm{Q}_{2}}}{2} .
$$

In order to obtain the Laplace inversion of (3.7), the following representations are made

$$
f_{1}(t)=\sin \alpha t, f_{2}(t)=\sin \beta t, \quad g_{1}(t)=e^{b_{1} t}, g_{2}(t)=e^{b_{2} t} .
$$

So that the Laplace inversion of (3.7) is the convolution of $f_{i}^{\prime} s$ and $g_{i}$ 's defined by

$$
f_{i} * g_{i}=\int_{0}^{t} f_{i}(t-u) g_{i}(u) d u, \quad i=1,2,3, \ldots
$$

Thus, Laplace inversion of (3.7) is given as

$$
\mathrm{Z}_{\mathrm{m}}(\mathrm{t})=\frac{\mathrm{Q}_{4}}{2\left(\mathrm{~b}_{1}-\mathrm{b}_{2}\right)}\left\{\mathrm{I}_{\mathrm{A}}-\mathrm{I}_{\mathrm{B}}-\mathrm{I}_{\mathrm{C}}+\mathrm{I}_{\mathrm{D}}\right\},
$$

where

$$
\begin{array}{ll}
I_{A}=\int_{0}^{t}(\sin \alpha t \cos \alpha u-\cos \alpha t \sin \alpha u) e^{b_{1} u} d u, & I_{B}=\int_{0}^{t}(\sin \alpha t \cos \alpha u-\cos \alpha t \sin \alpha u) e^{b_{2} u} d u, \\
I_{C}=\int_{0}^{t}(\sin \beta t \cos \beta u-\cos \beta t \sin \beta u) e^{b_{1} u} d u, & I_{D}=\int_{0}^{t}(\sin \beta t \cos \beta u-\cos \beta t \sin \beta u) e^{b_{2} u} d u .
\end{array}
$$


Evaluating the integrals (3.9), yields

$$
\begin{array}{ll}
I_{A}=\frac{1}{\alpha^{2}+b_{1}^{2}}\left\{\alpha e^{b_{1} t}-\alpha \cos \alpha t-b_{1} \sin \alpha t\right\}, & I_{B}=\frac{1}{\alpha^{2}+b_{2}^{2}}\left\{\alpha e^{b_{2} t}-\alpha \cos \alpha t-b_{2} \sin \alpha t\right\}, \\
I_{C}=\frac{1}{\beta^{2}+b_{1}^{2}}\left\{\beta e^{b_{1} t}-\beta \cos \beta t-b_{1} \sin \beta t\right\}, & I_{D}=\frac{1}{\beta^{2}+b_{2}^{2}}\left\{\beta e^{b_{2} t}-\beta \cos \beta t-b_{2} \sin \beta t\right\} .
\end{array}
$$

Thus, equation (3.8) in view of (3.9) leads to

$$
\begin{aligned}
Z_{m}(t)= & \frac{Q_{4}}{2\left(b_{1}-b_{2}\right)}\left\{\frac{\alpha e^{b_{1} t}-\alpha \cos \alpha t-b_{1} \sin \alpha t}{\alpha^{2}+b_{1}^{2}}-\frac{\alpha e^{b_{2} t}-\alpha \cos \alpha t-b_{2} \sin \alpha t}{\alpha^{2}+b_{2}^{2}}\right. \\
& \left.-\frac{\beta e^{b_{1} t}-\beta \cos \beta t-b_{1} \sin \beta t}{\beta^{2}+b_{1}^{2}}+\frac{\beta e^{b_{2} t}-\beta \cos \beta t-b_{2} \sin \beta t}{\beta^{2}+b_{2}^{2}}\right\} .
\end{aligned}
$$

Thus, in view of (3.3), taking into account (3.10) one obtains

$$
\begin{aligned}
W(x, t)= & \sum_{m=1}^{j} \frac{Q_{4}}{2\left(b_{1}-b_{2}\right)}\left\{\frac{\alpha e^{b_{1} t}-\alpha \cos \alpha t-b_{1} \sin \alpha t}{\alpha^{2}+b_{1}^{2}}-\frac{\alpha e^{b_{2} t}-\alpha \cos \alpha t-b_{2} \sin \alpha t}{\alpha^{2}+b_{2}^{2}}\right. \\
& \left.-\frac{\beta e^{b_{1} t}-\beta \cos \beta t-b_{1} \sin \beta t}{\beta^{2}+b_{1}^{2}}+\frac{\beta e^{b_{2} t}-\beta \cos \beta t-b_{2} \sin \beta t}{\beta^{2}+b_{2}^{2}}\right\} \cdot \sin \frac{m \pi x}{L},
\end{aligned}
$$

which represents the transverse response of structurally prestressed slender member to harmonic moving loads.

\section{Comments on the closed-form solution}

Occurrence of resonance in a dynamical system is of a great concern in design engineering and engineering analysis as the amplitude of vibration of a structural member carrying travelling loads may grow without bound. Thus, this section seeks to examine and establish the conditions under which this unpleasant phenomenon may occur. It is clearly seen from equation (3.11) that a structurally damped system considered in this study will experience a state of resonance under any of the following stated conditions:

$$
\begin{array}{ll}
\text { i. } & b_{1} \alpha^{2}+b_{1}^{3}=b_{2} \alpha^{2}+b_{2} b_{1}^{2}, \\
\text { ii. } & b_{2} \alpha^{2}+b_{2}^{3}=b_{1} \alpha^{2}+b_{1} b_{2}^{2}, \\
\text { iii. } & b_{1} \beta^{2}+b_{1}^{3}=b_{2} \beta^{2}+b_{2} b_{1}^{2}, \\
\text { iv. } & b_{2} \beta^{2}+b_{2}^{3}=b_{1} \beta^{2}+b_{1} b_{2}^{2}
\end{array}
$$

and the velocity, at which this phenomenon may occur termed the critical velocity associated with the conditions listed in (4.1) respectively are given as

$$
\begin{aligned}
& u_{c r}^{1}=\frac{L}{2 \pi k} \sqrt{8 Q_{2}+2 \sqrt{Q_{1}^{2}-4 Q_{2}-3 Q_{1}^{2}}}-\frac{\omega L}{k \pi}, \quad u_{c r}^{2}=\frac{L}{2 \pi k} \sqrt{8 Q_{2}-2 \sqrt{Q_{1}^{2}-4 Q_{2}-3 Q_{1}^{2}}-\frac{\omega L}{k \pi}} \\
& u_{c r}^{3}=\frac{\omega L}{k \pi}-\frac{L}{2 \pi k} \sqrt{8 Q_{2}+2 \sqrt{Q_{1}^{2}-4 Q_{2}-3 Q_{1}^{2}}}, \quad u_{c r}^{4}=\frac{\omega L}{k \pi}-\frac{L}{2 \pi k} \sqrt{8 Q_{2}-2 \sqrt{Q_{1}^{2}-4 Q_{2}-3 Q_{1}^{2}}}
\end{aligned}
$$

where all the parameters are as previously defined. 


\section{Numerical Result and discussion}

In this section, the analysis proposed in the previous sections are illustrated by considering a homogeneous beam of modulus of elasticity $E=2.9012 \times 10^{9} \mathrm{~N} / \mathrm{M}^{2}$, the moment of inertial $\mathrm{I}=2.87698 \times$ $10^{-3} \mathrm{kgm}^{2}$, the beam span $\mathrm{L}=12.192 \mathrm{~m}$ and the mass per unit length of the beam $\mu=2758.291 \mathrm{~kg} / \mathrm{m}$. The load is also assumed to travel along the beam with constant velocity $V=3.128 \mathrm{~m} / \mathrm{s}$, the values of foundation moduli $\mathrm{K}_{0}$ are varied between $0 \mathrm{~N} / \mathrm{m}^{3}$ and $4 \times 10^{8} \mathrm{~N} / \mathrm{m}^{3}$, the values of axial force $\mathrm{N}$ varied between $0 \mathrm{~N}$ and $2.0 \times 10^{11} \mathrm{~N}$. The values of the shear modulus $\mathrm{G}_{0}$ are varied between $0 \mathrm{~N} / \mathrm{m}^{3}$ and $4 \times 10^{8} \mathrm{~N} / \mathrm{m}^{3}$ and the values of the rotatory inertia correction factor $r^{0}$ varied between $0 \mathrm{~N} / \mathrm{m}^{3}$ and $5.5 \times 10^{5} \mathrm{~N} / \mathrm{m}^{3}$.

Figure 1 displays the transverse displacement response of a simply supported uniform beam under the action of harmonic forces traveling at constant velocity for the various values of axial force $\mathrm{N}$ and for fixed values of subgrade moduli $\mathrm{F}_{0}=40000$ and shear modulus $\mathrm{G}_{0}=30000$ and rotatory inertia $\mathrm{r}^{0}=0.5$. The figures show that as $\mathrm{N}$ increases, the response amplitude of the uniform beam decreases. For various traveling time $t$, the displacement response of the beam for various values of subgrade moduli $F_{0}$ and for fixed values of axial force $N=20000$, shear modulus $G_{0}=30000$ and $r^{0}=0.5$ are shown in Figure 2. It is observed that the higher the values of subgrade moduli $F_{0}$ the smaller the response amplitude of the vibrating beam. Figure 3 displays the deflection profile of the simply supported uniform beam under harmonic forces traveling at constant velocity for various values of shear modulus $\mathrm{G}_{0}$ and fixed values of axial force $N=20000$, subgrade moduli $F_{0}=40000$ and $r^{0}=0.5$. It is seen from the figure that as the values of the shear modulus increases the deflection of the beam decreases significantly. The response of the elastic beam to the traveling harmonic forces for various values of the load position coordinate $x$ and for fixed values of other parameters is displayed in Figure 4. It is deduced from the figure that the dynamic deflection at the mid-span of the beam is very large compare to other load positions. Figure 5 displays the deflection profile of the simply supported uniform beams subjected to forces traveling at constant velocity for various values of rotatory inertial correction factor $r^{0}$ and fixed values of axial force $\mathrm{N}=20000$, subgrade moduli $\mathrm{F}_{0}=40000$ and shear modulus $\mathrm{G}_{0}=30000$. The figure clearly shows that as the value of the rotatory inertia $r^{0}$ increases the deflection of the simply supported uniform beam under the action of moving forces traveling at constant velocity decreases.

Figures 6 and 7 depict the response amplitude of a simply supported uniform beam under the action of harmonic forces traveling at constant velocity for various values of external and internal damping $D_{d}$ and $D_{i}$ and for fixed values of axial force $N=20000$, subgrade moduli $F_{0}=40000$ and shear modulus $\mathrm{G}_{0}=40000$. The figures show that higher values of these parameters reduce the deflection of the beam considerably. For various values of circular frequency $\omega$ and for fixed values of other parameters, Figure 8 depicts the deflection profile of the vibrating beam. The figure clearly shows that the higher the value of the circular frequency the lower the deflection of the beam. The transverse response of the elastic uniform beam to the traveling load for various load velocities is presented in Figure 9. It is shown from the figure that the higher the speed of the traveling load the larger the deflection of the structural member.

\section{Concluding remarks}

The problem of the response of elastic beam carrying traveling variable magnitude load is investigated in this study. Solution procedure, involving assumed mode method and integral transform method is developed to obtain exact solution to the fourth order partial differential equation describing the motion of the beam-load system. Various results in plotted curves show that, the presence of some vital structural parameters such as the axial force $\mathrm{N}$, rotatory inertia correction factor $r^{0}$, the foundation modulus $\mathrm{F}_{0}$ and the shear modulus $G_{0}$ significantly enhances the stability of the beam when under the actions of the fast traveling load. Conditions under which the beam-load system will experience resonance phenomenon are also established. The speeds at which this may occur are also established. 


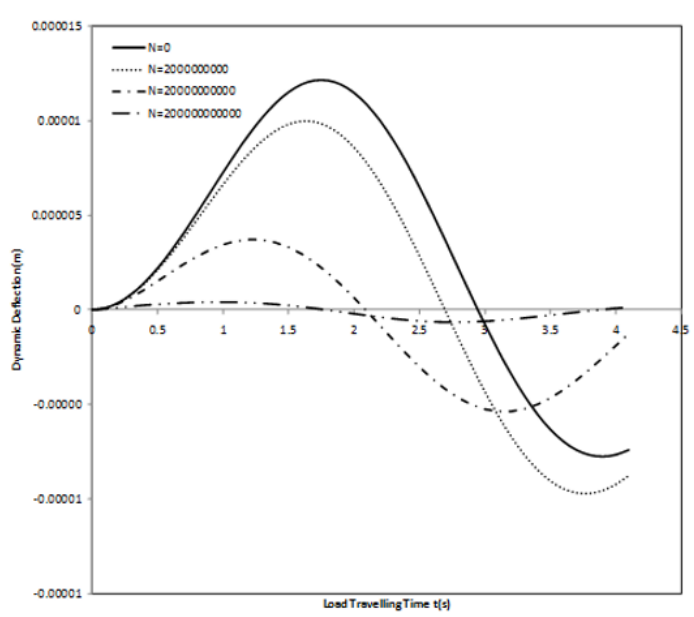

Figure 1: Transverse displacement response of a simply supported structural members resting on elastic foundation and under the actions of uniform partially distributed forces for various values of axial force $\mathrm{N}$ and for fixed values of $\mathrm{F}_{0}=40000, \mathrm{G}_{0}=30000$.

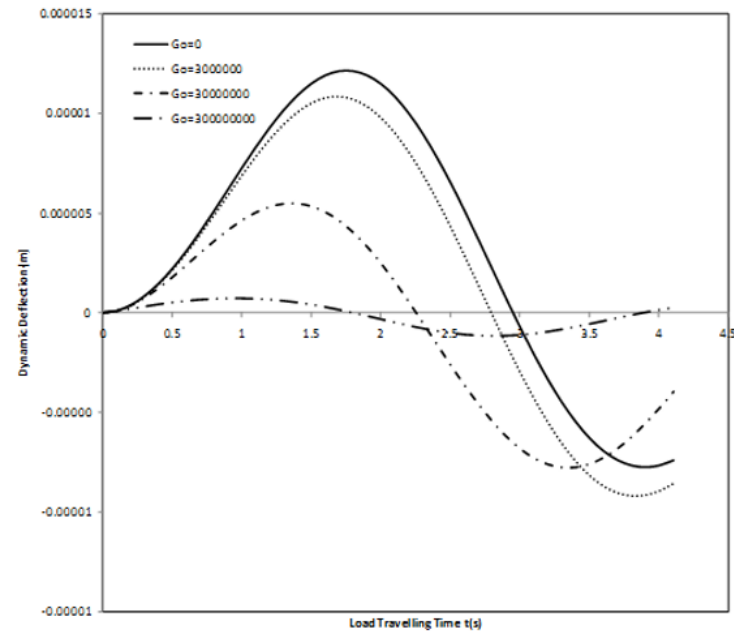

Figure 3: Transverse displacement response of a simply supported structural members resting on elastic foundation and under the actions of uniform partially distributed forces for various values of axial force $G_{0}$ and for fixed values of $\mathrm{F}_{0}=40000, \mathrm{~K}_{0}=30000$.

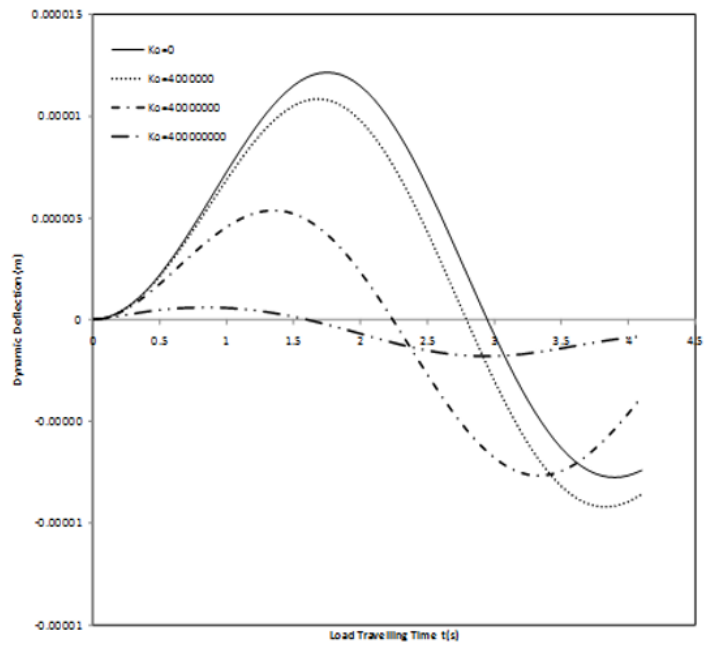

Figure 2: Transverse displacement response of a simply supported structural members resting on elastic foundation and under the actions of uniform partially distributed forces for various values of axial force $\mathrm{K}_{0}$ and for fixed values of $\mathrm{F}_{0}=40000, \mathrm{G}_{0}=30000$.

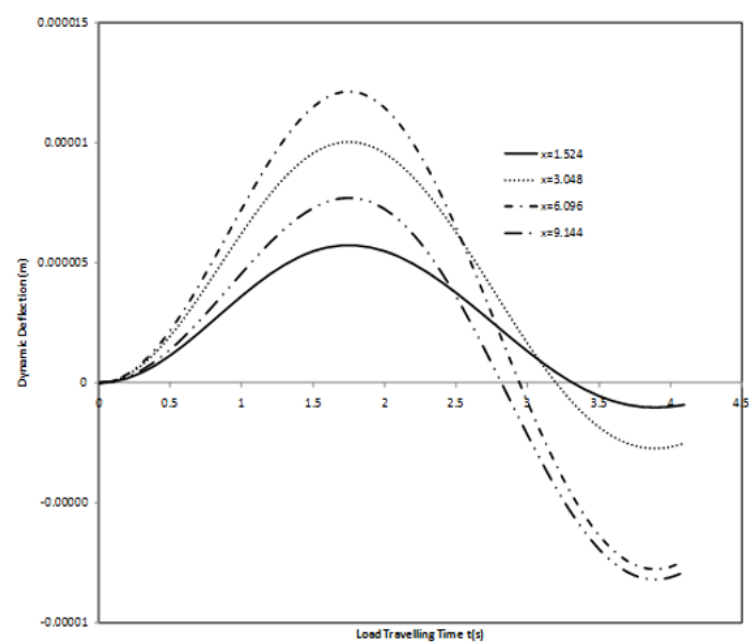

Figure 4: Response Amplitude of a simply supported structural members resting on elastic foundation and under the actions of uniform partially distributed forces for various values of the load position and for fixed values of $\mathrm{G}_{0}=30000, \mathrm{~F}_{0}=40000$ and $\mathrm{N}=20000$. 


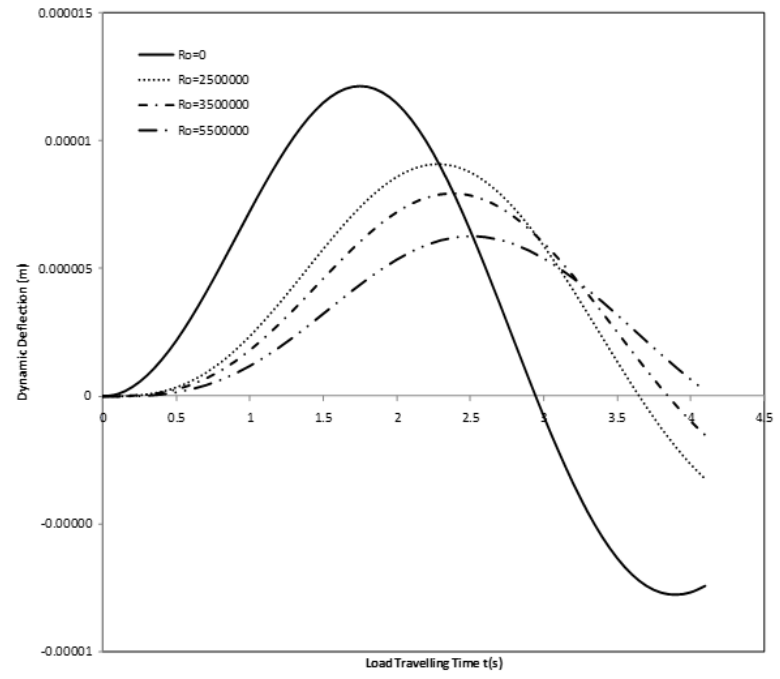

Figure 5: Response Amplitude of a simply supported structural members resting on elastic foundation and under the actions of uniform partially distributed forces for various values of the rotatory inertial $\mathrm{r} 0$ and for fixed values of $\mathrm{G}_{0}=30000, \mathrm{~F}_{0}=40000$ and $\mathrm{N}=20000$.

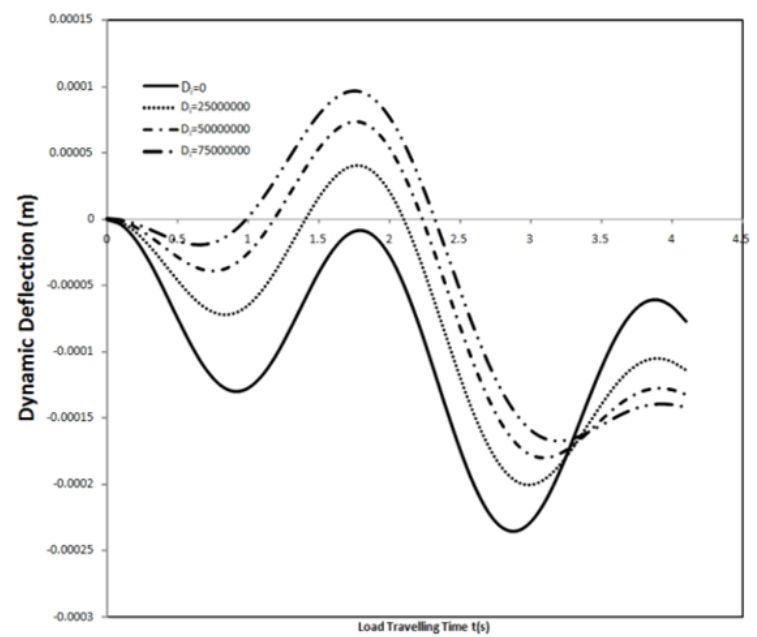

Figure 7: Response of a simply supported structural members resting on elastic foundation to uniform partially distributed forces for various values of internal damping $D_{i}$ and for fixed values of $\mathrm{G}_{0}=30000, \mathrm{~F}_{0}=40000$ and $\mathrm{N}=20000$.

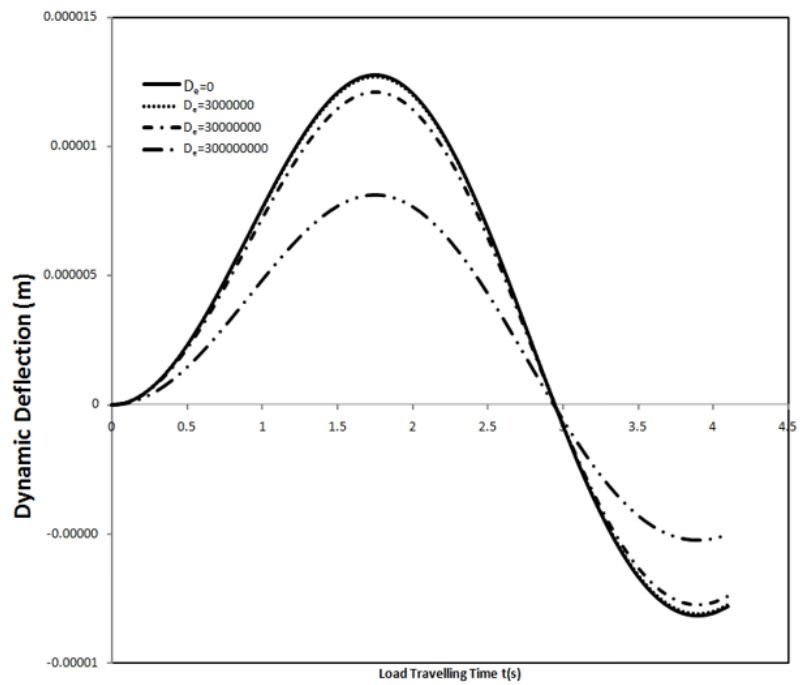

Figure 6: Response of a simply supported structural members resting on elastic foundation to uniform partially distributed forces for various values of external damping $\mathrm{D}_{e}$ and for fixed values of $\mathrm{G}_{0}=30000, \mathrm{~F}_{0}=40000$ and $\mathrm{N}=$ 20000.

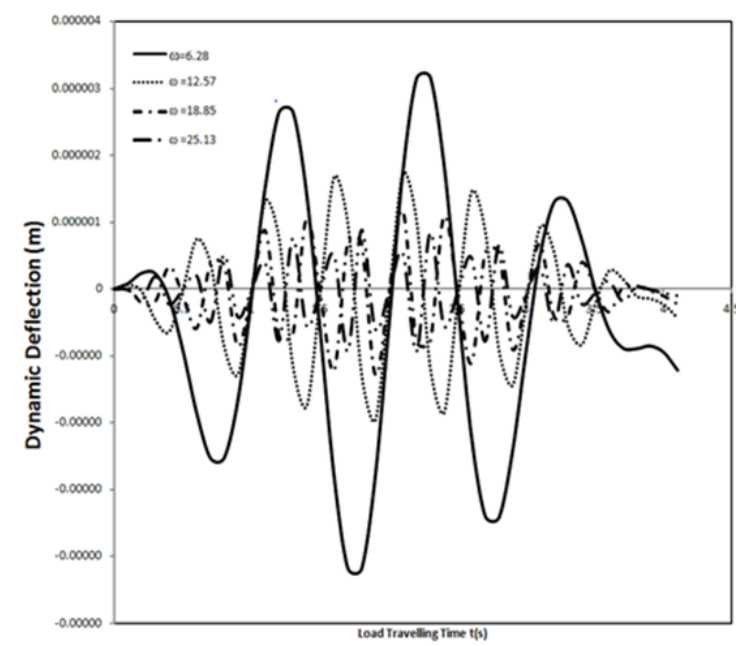

Figure 8: Response Amplitude of a simply supported structural members resting on elastic foundation and under the actions of uniform partially distributed forces for various values of the load circular fequency $\omega$ and for fixed values of $\mathrm{G}_{0}=30000, \mathrm{~F}_{0}=40000$ and $\mathrm{N}=20000$. 


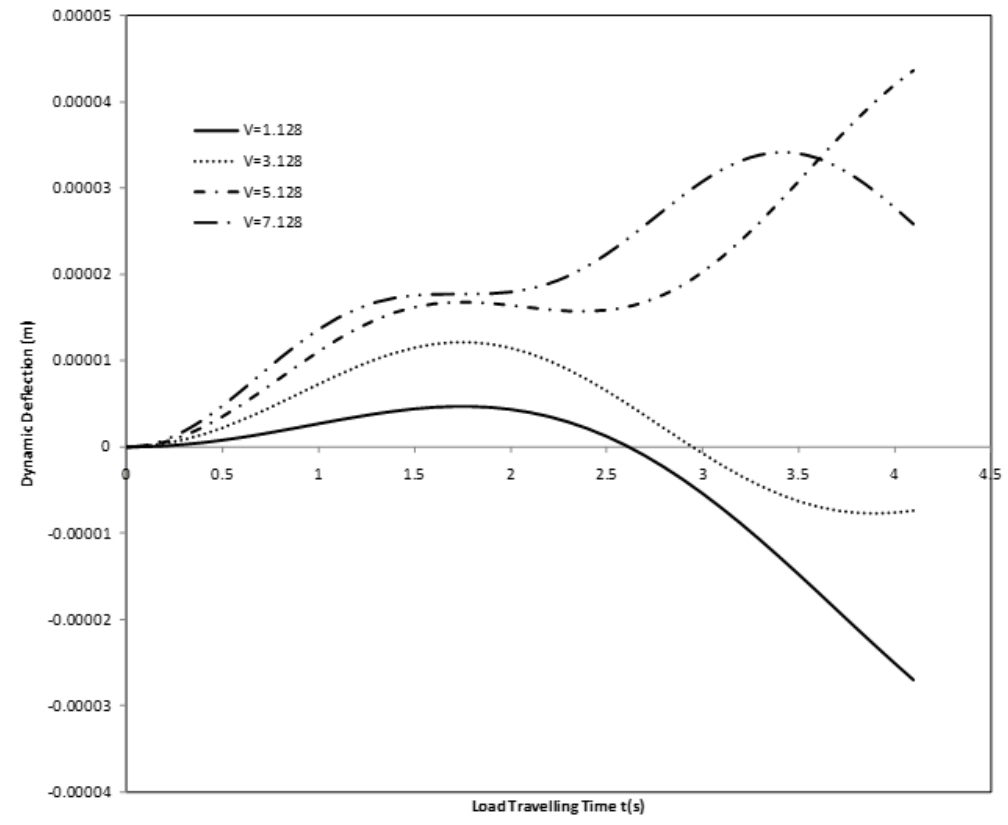

Figure 9: Response Amplitude of a simply supported structural members resting on elastic foundation and under the actions of uniform partially distributed forces for various values of the load velocity and for fixed values of $\mathrm{G}_{0}=30000, \mathrm{~F}_{0}=40000$ and $\mathrm{N}=20000$.

\section{References}

[1] M. Abu-Hilal, H. Zibdeh, Vibration analysis of beams with general boundary conditions traversed by a moving force, J. Sound Vibration, 229 (2000), 377-388. 1

[2] G. G. Adams, Critical speeds and the response of a tensioned beam on an elastic foundation to repetitive moving loads, Int. J. Mech. Sci., 37 (1995), 773-781. 1

[3] Y. Araar, B. Radjel, Delection Analysis of Clamped Rectangular Plates of Variable Thickness on Elastic Foundation by the Galerkin Method, Res. J. Appl. Services, 2 (2007), 1077-1082. 1

[4] T. O. Awodola, Variable velocity influence on the vibration of simply supported bernoulli-euler beam under exponentially varying magnitude moving load, J. Math. Stat., 3 (2007), 228-232. 1

[5] T. O Awodola, B. Omolofe, Response to concentrated moving masses of elastically supported rectangular plates resting on winkler elastic foundation, J. Theoretical Appl. Mech., 44 (2014), 65-90.

[6] R. S. Ayre, L. S. Jacobsen, C. S. Hsu, Transverse vibration of one-and of two-span beams under the action of a moving mass load, Proceedings of the first U. S. National Congress of Applied Mechanics, 1951 (1951), 81-90. 1

[7] C. Bilello, L. A. Bergman, Vibration of damaged beams under a moving mass: Theory and experimental validation, J. Sound Vibration, 274 (2004), 567-582. 1

[8] J. Clastornic, M. Eisenberger, D. Z. Yankelevsky, M. A. Adin, Beams on Variable Winkler Elastic Foundation, J. Appl. Mech., 53 (1986), 925-928. 1

[9] E. Esmailzadeh, M. Ghorashi, Vibration analysis of beams traversed by moving masses, Intern. J. Engin., 8 (1995), 213-220. 1

[10] E. Esmailzadeh, M. Ghorashi, Vibration analysis of beams traversed by uniform partially distributed moving masses, J. Sound Vibration, 184 (1995), 9-17. 1

[11] L. Frýba, Vibration of solids and structures under moving loads, Springer Science \& Business Media, Groningen, (1972). 1

[12] M. H. Ghayesh, S. E. Khadem, Rotatory inertia and temperature effects on non-linear vibration, steady-state response and stability of an axially moving beam with time-dependent velocity, Int. J. Mech. Sci., 50 (2008), 389-404. 1

[13] T. R. Hamada, Dynamic analysis of a beam under a moving force: a double laplace transform solution, J. Sound Vibration, 74 (1981), 221-233. 1

[14] M. H. Hsu, Vibration characteristics of rectangular plates resting on elastic foundations and carrying any number of sprung masses, Int. J. Appl. Sci. Eng., 4 (2006), 83-89. 1

[15] T. C. Huang, V. N. Shah, Elastic system moving on an elastically supported beam, J. Vibration Acoustics Stress Reliab. Design, 106 (1984), 292-297. 1 
[16] Y. H. Lin, Comments on vibration analysis of beams traversed by uniform partially distributed moving masses, J. Sound Vibration, 199 (1997), 697-700. 1

[17] A. V. Metrikine, S. N. Verichev, J. Blaauwendraad, Stability of a two-mass oscillator moving on a beam supported by a visco-elastic half-space, Int. J. Solid Structures, 42 (2005), 1187-1207. 1

[18] G. Muscolino, A. Palmeri, Response of beams resting on viscoelastically damped foundation to moving oscillators, Int. J. Solid Srtuctures, 44 (2007), 1317-1336. 1

[19] D. K. Nguyen, Free vibration of prestressed Timoshenko beams resting on elastic foundation, Vietnam J. Mech., 29 (2007), 1-12. 1

[20] M. Olsson, On the fundamental moving load problem, J. Sound Vibration, 145 (1991), 299-307.

[21] B. Omolofe, Deflection profile analysis of beams on two-parameter elastic subgrade, Latin Amer. J. Solid Structures, 10 (2013), 263-282. 1

[22] B. Omolofe, A. Adedowole, Response characteristics of non-uniform beam with time-dependent boundary conditions and under the actions of travelling distributed masses, J. Appl. Math. Comput. Mech., 16 (2017), 77-99. 1

[23] B. Omolofe, T. O. Adeloye, Behavioral study of finite beam resting on elastic foundation and subjected to travelling distributed masses, Latin Amer. J. Solids Structures, 14 (2017), 312-334. 1

[24] B. Omolofe, S. N. Ogunyebi, Transverse vibrations of elastic thin beam resting on variable elastic foundations and subjected to traveling distributed forces, Pacific J. Sci. Tech., 10 (2009), 112-119. 1

[25] M. Ouchenane, R. Lassoued, K. Ouchenane, Vibration analysis of bridges structures under the influence of moving loads, (a conference proceeding), 3rd International conference on integrity, reliability and failure, Porto/Portugal, (2009), 20-24. 1

[26] A. V. Presterev, L. A. Bergman, An improved series expansion of the solution to the moving oscillator problem, J. Vib. Acoust., 122 (2000), 54-61. 1

[27] A. V. Presterev, L. A. Bergman, C. A. Tan, T.-C. Tsao, B. Yang, On the asymptotics of the solution of the moving oscillator problem, J. Sound Vibration, 260 (2003), 519-536. 1

[28] G. V. Rao, Linear dynamics of an elastic beam under moving loads, J. Vib. Acoust., 122 (2000), 281-289. 1

[29] B. J. Ryu, Dynamic analysis of a beam subjected to a concentrated moving mass, Master Thesis (Yonsei University), Seoul, (1983). 1

[30] S. Sadiku, H. H. E. Leipholz, On the dynamics of elastic systems with moving concentrated masses, Ing. Arch., 57 (1987), 223-242. 1

[31] D. Stǎncioiu, H. Ouyang, J. E. Mottershead, Vibration of a beam excited by a moving oscillator considering separation and reattachment, J. Sound Vibration, 310 (2008), 1128-1140. 1

[32] M. M. Stanişić, J. A. Euler, S. T. Montgomery, On a theory concerning the dynamical behaviour of structures carrying moving masses, Ing. Arch., 43 (1974), 295-305. 1

[33] M. M. Stanişić, J. C. Hardin, On the response of beams to an arbitrary number of concentrated moving masses, J. Franklin Ins., 287 (1969), 115-123. 1

[34] G. G. Strokes, Discussion of a differential equation relating to the breaking of railway bridges, Transactions of the Cambridge Philosophical Society, 85 (1849), 707-735. 1

[35] D. Thambiratnam, Y. Zhuge, Dynamic analysis of beams on an elastic foundation subjected to moving loads, J. Sound Vibration, 198 (1996), 149-169.

[36] S. P. Timoshenko, On the transverse vibrations of bars of uniform cross-section, The London, Edinburgh, and Dublin Philosophical Magazine and Journal of Science, 43 (1922), 125-131. 1

[37] Y. M. Wang, The dynamical analysis of a finite inextensible beam with an attached accelerating mass, Int. J. Solid Structures, 35 (1998), 831-854.

[38] R. T. Wang, T. H. Chou, Nonlinear vibration of timoshenko beam due to a moving force and the weight of beam, J. Sound Vibration, 218 (1998), 117-131. 1

[39] J. J. Wu, Vibration analyses of a portal frame under the action of a moving distributed mass using moving mass element, Int. J. Num. meth. Engin., 62 (2005), 2028-2052. 1

[40] J. Ying, C. F. Lu, W. Q. Chen, Two-dimensional elasticity solutions for functionally graded beams resting onelastic foundations, Composite Structures, 84 (2008), 209-219. 1

[41] D. M. Yoshida, W. Weaver, Finite element analysis of beams and plates with moving loads, Int. Associat. Bridge Structural Eng., 31 (1971), 179-195. 1 